\title{
LA POLINIZACIÓN NATURAL EN EL MARACUYÁ (Passiflora edulis f. flavicarpa Degener) COMO UN SERVICIO REPRODUCTIVO Y ECOSISTÉMICO ${ }^{1}$
}

\author{
Juan Carlos Arias-Suárez ${ }^{2}$, John Albeiro Ocampo-Pérez ${ }^{3}$, Ramiro Urrea-Gómez ${ }^{4}$
}

\begin{abstract}
RESUMEN
La polinización natural en el maracuyá (Passiflora edulis f. flavicarpa Degener) como un servicio reproductivo y ecosistémico. El objetivo de esta investigación fue estudiar los mecanismos de la polinización en el cultivo del maracuyá y la importancia de los polinizadores naturales. El estudio se realizó en los años 2010-2011, en el municipio de Palestina, Caldas, Colombia a $1050 \mathrm{msnm}$. Se emplearon cinco tratamientos de polinización: natural (P.N), manual dirigida (P.M.D), autopolinización manual y espontánea (A.P, A.P.E) y geitonogamia (G), en 48 accesiones de maracuyá. Los resultados mostraron diferencias significativas entre tratamientos, la P.N realizada por abejorros del género Xylocopa spp. obtuvo el mayor porcentaje de frutos formados $(88 \%)$, seguido por P.M.D (60\%), y solo un $3 \%$ en los tratamientos de autopolinización (A.P.E, A.P, G). De igual manera, la P.N presentó mayor peso promedio del fruto (203 g) y porcentaje de pulpa más semilla $(47 \%)$ en comparación a P.M.D (187,5 g y 44,7\%). La actividad y efectividad de los polinizadores estuvo correlacionada con la temperatura máxima diurna $(\mathrm{r}=0,69)$, brillo solar $(\mathrm{r}=0,56)$ y humedad relativa $(\mathrm{r}=0,64)$. Cuando las dos primeras variables descendieron simultáneamente por debajo de $26^{\circ} \mathrm{C}$ y menos de tres horas por día, se alcanzaron los mayores porcentajes de fructificación.
\end{abstract}

Palabras clave: relación planta/polinizador, Xylocopa spp, fruta tropical, abeja carpintera.

\begin{abstract}
The natural pollination in yellow passion fruit (Passiflora edulis f. flavicarpa Degener) as a reproductive and ecosystem service. The goal of this research was to study the mechanism of pollination in the yellow passion fruit and to identify natural pollinators of importance. The study was carried out in 2010-2011 in the Palestina (Caldas) to 1050 masl, by performing five treatments of pollination: natural (P.N), hand pollination (P.M.D), spontaneous and assisted self-pollination (A.P, A.P.E.) and geitonogamy/autogamy (G) in 48 accessions of yellow passion fruit. The results showed significative differences between treatments, the highest percentage of set fruit (88\%) was observed during P.N carried by bumblebees from genus Xylocopa spp, followed by P.M.D. $(60 \%)$. In contrast self-pollination treatments (A.P.E, A.P, G) achieved only a 3\% set fruit. Likewise, the P.N showed the highest fruit weight average (203 g), and the highest flesh to seed ratio (47\%), followed closely by P.M.D (187.5 $\mathrm{g}$ and $44.7 \%$, respectively). The activity and effectiveness of pollinators showed a correlation with the maximum daytime temperature $(\mathrm{r}=0.69)$, solar irradiance $(\mathrm{r}=0.56)$ and relative humidity $(\mathrm{r}=0.64)$. However, the highest percentage of fruiting was achieved when the first two environmental variables decreased simultaneously below $26^{\circ} \mathrm{C}$ and three hours per day less.
\end{abstract}

Keywords: plant/pollinator relationship, Xylocopa spp, tropical fruit, carpenter bee.

\footnotetext{
Recibido: 18 de abril, 2013. Aceptado: 18 de marzo, 2014. Parte del trabajo de grado para optar al título de Ingeniero Agrónomo del primer autor. Facultad de Ciencias Agropecuarias, Universidad de Caldas, Colombia.

2 Universidad Nacional de Colombia sede Palmira, Facultad de Ciencias Agropecuarias, A.A. 237 Palmira, Colombia. jucariassu@unal.edu.co

3 Universidad Nacional de Colombia sede Palmira/Centro Internacional de Agricultura Tropical - CIAT, A.A. 237 Cali, Colombia. jaocampop@ unal.edu.co

${ }^{4}$ Universidad de Caldas, Facultad de Ciencias Agropecuarias, A.A 275 Manizales, Colombia. ramiro.urrea@ucaldas.edu.co
}

CC (1) $\Theta(C) 2014$ Agronomía Mesoamericana es desarrollada en la Universidad de Costa Rica y se encuentra licenciada con Creative Commons Reconocimiento-NoComercial-SinObraDerivada 3.0 Costa Rica. Para más información escríbanos a pccmca@ucr.ac.cr 


\section{INTRODUCCIÓN}

El género Passiflora L. es el más importante de la familia Passifloraceae, con cerca de 80 especies con fruto comestible y distribuidas en la zona neotropical desde el nivel del mar hasta los 3800 msnm (Coppens d'Eeckenbrugge et al., 1997). El maracuyá (Passiflora edulis f. flavicarpa Degener) es la principal especie del género, debido a que sus frutos son comercializados en mercados nacionales e internacionales como fruta fresca y procesada (Lima y Cunha, 2004). Brasil es el centro de origen del maracuyá y es cultivado en zonas tropicales en cuatro continentes. Los principales productores a nivel mundial son Brasil, Ecuador, Colombia y Perú con aproximadamente 805000 t/año (Passionfruit, 2011).

Esta fruta fue introducida en Colombia a inicio de los años 60`s y actualmente existen cerca de 5800 ha de cultivo, con una producción de 90000 t, de las cuales el $70 \%$ son destinados a la industria como jugo concentrado para exportación, principalmente a Holanda y Puerto Rico (Agronet, 2012). A nivel nacional, en los departamentos del Huila, Valle del Cauca y Meta se concentra la mayor producción con cerca de 2600 ha y con rendimientos promedios que varían entre 15 y $20 \mathrm{t} / \mathrm{ha} / \mathrm{año}$. Debido a lo anterior, este cultivo provee gran cantidad de empleos, con aproximadamente 240 jornales/ha/año, de los cuales el 33\% son destinados a la recolección (Jaramillo et al., 2009).

El maracuyá es una especie diploide $(2 n=18)$ con flores hermafroditas y un alto grado de autoincompatibilidad (Suassuna et al., 2000). La polinización es cruzada (alogamia) y realizada principalmente por insectos de género Xylocopa, conocidos comúnmente como abejorros (González et al., 2009). Estudios realizados en Brasil y el sur de Estados Unidos (Florida) indican que la autoincompatibilidad puede variar entre $80 \mathrm{y}$ 100\% (Akamine y Girolami, 1959; Knight y Winters, 1962). Por otro lado, Suassuna et al. (2000) mencionan que el fenómeno de autoincompatibilidad en maracuyá es controlado por la presencia de genes esporofíticos que actúan en asociación con genes gametofíticos.

En el género Passiflora L., la evolución de diferentes colores, la presencia o ausencia de aromas atrayentes y la posición de la corona con respecto al androginóforo, han jugado un papel importante en la especiación y la asociación con diferentes polinizadores como insectos, aves y mamíferos (Ramírez, 2006).
En el trópico, el 70\% de los cultivos dependen de la acción de polinizadores para su producción (Roubik, 1995); por lo que son considerados como un servicio reproductivo y ecosistémico (Calle et al., 2010). El flujo genético en estas especies depende de vectores que transporten el polen hasta el estigma para la fecundación y producción de frutos (Bos et al., 2007; Siqueira et al., 2009; Rendón et al., 2013).

El maracuyá posee varias recompensas para los insectos polinizadores, los cuales son atraídos por los colores vistosos de la flor, aromas fuertes y abundancia de néctar (Siqueira et al., 2009). En esta especie se ha reportado diferentes polinizadores de Xylocopa spp. como los responsables de la polinización e intercambio genético en los cultivos comerciales (Peña, 2003; González et al., 2009). Los abejorros polinizadores (Xylocopa spp.) construyen sus nidos dentro de los postes de madera que soportan la planta o en troncos de árboles aledaños a los cultivos (Ruggiero et al., 1996; González et al., 2009).

Las poblaciones de abejorros dependen principalmente de la abundancia de néctar (Akamine et al., 1954), del estado de conservación de los hábitat alrededor de los cultivos (Calle et al., 2010) y de las condiciones climáticas de cada zona (Da Silva et al., 1999). Además, el uso excesivo e inadecuado de agroquímicos y el desconocimiento de su importancia en los agroecosistemas, ha causado una reducción de las poblaciones de Xylocopa spp., lo que ha traído como consecuencia una disminución en la producción de frutos en el cultivo de maracuyá (Calle et al., 2010). No obstante, cuando las poblaciones de los polinizadores es baja, los productores realizan la polinización manual, por medio de la extracción del polen de las anteras de una flor y llevándolo a los estigmas de otra (Lima y Cunha, 2004). Esta práctica de polinización puede mejorar los porcentajes en la formación de frutos entre 50\% y 87\% (Bos et al., 2007; Siqueira et al., 2009). Además, con el uso de esta metodología, los frutos producidos son de mayor tamaño en relación con los obtenidos por polinización natural (Akamine y Girolami, 1959). En contraste, Ángel-Coca et al. (2011) indican que la participación de los insectos polinizadores en P. edulis f. edulis Sims incrementa características del fruto, debido a la probabilidad de obtener un mayor número de óvulos fecundados. En adición, Silveira et al. (2012) muestran que no existen diferencias en la producción de frutos cuando las flores 
son polinizadas manualmente o naturalmente por insectos de Xylocopa (X. frontalis Oliver y X. grisescens Lepeletier).

Las percepciones de los agricultores acerca de la biología de la polinización y el servicio que prestan estos insectos en los cultivos no es clara ni valorada (Ghazoul, 2007). El valor económico per se del servicio de polinización por insectos pierde importancia cuando los productores toman en cuenta los riesgos inherentes a la producción agrícola y en especial en la protección del cultivo (Calle et al., 2010).

En Colombia, la polinización natural de maracuyá es más frecuente que la manual, debido a que la mayoría de las plantaciones se localizan cerca del bosque natural o semi-natural. En el departamento del Valle del Cauca los agricultores emplean la polinización manual para complementar la polinización por insectos (Calle et al., 2010). Sin embargo, en muchas plantaciones de otros departamentos (e.g. Tolima y Huila) esta práctica se realiza debido a un mayor uso de agroquímicos, sin tener en cuenta los servicios que ofrecen la polinización natural y la protección del medio ambiente.

El objetivo de esta investigación fue estudiar los mecanismos de la polinización en el cultivo del maracuyá y la importancia de los polinizadores naturales en una zona agroecológica en Colombia. Además, resaltar la eficiencia de los insectos polinizadores para que los productores adopten el concepto de servicios de polinización, que justifique la conservación de estos organismos en los agroecosistemas.

\section{MATERIALES Y MÉTODOS}

\section{Área de estudio}

La investigación se llevó a cabo en la granja Luker, localizada en el municipio de Palestina, Caldas, Colombia a $1023 \mathrm{msnm}\left(5^{\circ} 4\right.$ ' 25,95' N, - 75 41' 4,71'" $\mathrm{W})$ en un ecosistema de bosque húmedo premontano (bh-PM). Las condiciones climáticas presentes en la zona fueron: temperatura media de $23^{\circ} \mathrm{C}$, precipitación promedio anual de $2200 \mathrm{~mm}$, humedad relativa de $78 \%$ y brillo solar de $2200 \mathrm{~h} / \mathrm{luz} / \mathrm{año.} \mathrm{Los} \mathrm{principales}$ sistemas agrícolas presentes en el área de estudio son cacao (Theobroma cacao L.), papaya (Carica papaya L.), plátano (Musa AAB), maíz (Zea mays L.) y otras pasifloras como $P$. maliformis L., $P$. alata Curtis, $P$. caerulea L. y P. cincinnata Mast.

\section{Material vegetal}

El estudio se llevó a cabo en la Colección Nacional de Maracuyá (P. edulis f. flavicarpa) desde octubre de 2010 hasta julio de 2011. La colección está constituida por 52 accesiones, provenientes de Colombia (47), Ecuador (3), Brasil (1) y Costa Rica (1) donde cada accesión está representada por diez plantas, sembradas a una distancia de $3 \mathrm{~m}$ entre surcos y $3 \mathrm{~m}$ entre plantas bajo el sistema de conducción en espaldera sencilla.

\section{Estudio biología de la reproducción}

Los ensayos de polinización natural, autopolinización asistida y manual dirigida (Figura 1) se realizaron en diferentes épocas de floración, teniendo en cuenta que la apertura de la flor o antesis en la zona de estudio ocurre entre las 11:30 am y 1:00 pm. La metodología seguida en esta investigación fue la desarrollada por Ángel-Coca et al. (2011) en P. edulis f. edulis, bajo diferentes tratamientos, donde se evaluó el efecto de estos sobre la producción de frutos, características físicas y número de semillas:

Tratamiento I (Polinización Natural= P.N): Se marcaron flores antes de la antesis y se dejaron expuestas a la acción de los insectos polinizadores. Los insectos vectores fueron capturados (16) y fotografiados (Figura 1a) e identificados con las claves taxonómicas de González et al. (2009) y determinados como especies del género Xylocopa spp. (Hymenotera: Apidae: Xylocopini). La abundancia de polinizadores se determinó con base en la metodología de Da silva et al. (1999), donde se considera el número de visitas del polinizador a cada flor durante el periodo de antesis (cinco horas).

Tratamiento II (Polinización Manual Dirigida= P.M.D): Se marcaron y embolsaron botones florales (en bolsa de muselina 10x20 cm), en el momento de la apertura floral fueron emasculados y polinizados manualmente con polen proveniente de flores de otras plantas. Una vez realizada la polinización la flor se embolsó nuevamente.

Tratamiento III (Autopolinización Espontánea= A.P.E): Se marcaron y embolsaron botones florales 

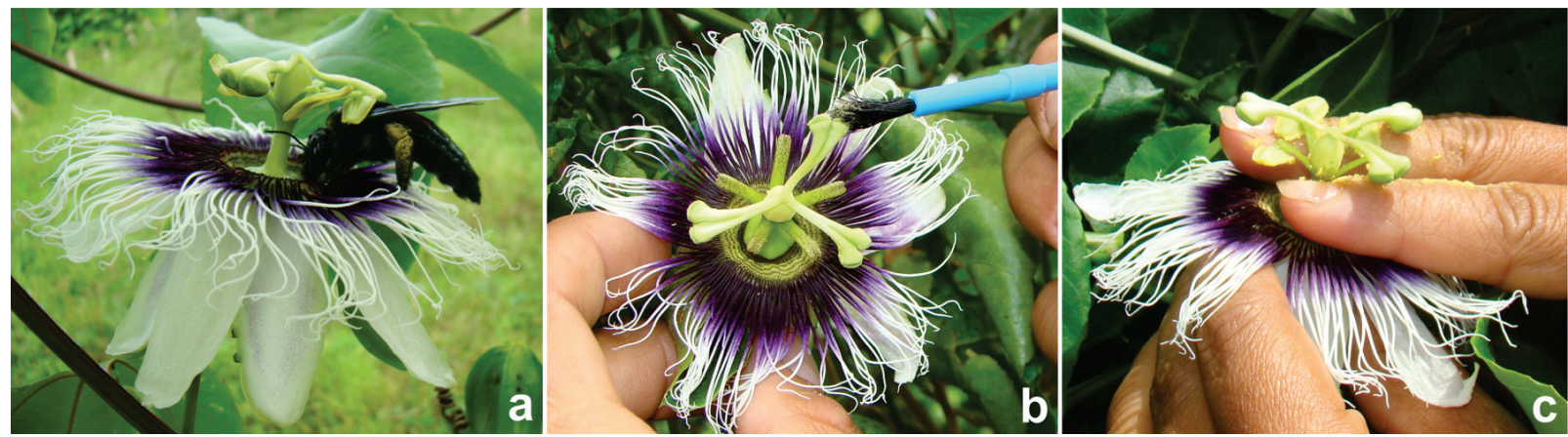

Figura 1. Tipos de polinización en maracuyá: a. natural por el abejorro Xylocopa spp.; b. asistida y c. manual dirigida, empleada por los productores. Granja Luker. Colombia. 2010-2011.

(en bolsa de muselina 10x20 cm), evitando la visita de los agentes polinizadores. De esta forma, la flor se fecundó naturalmente por sus propios mecanismos. Las flores seleccionadas presentaban estigmas totalmente curvos (por debajo de las anteras), debido a que este tipo de flor favorece la autopolinización y de esta manera se evita el sesgo con la presencia de los otros tipos de flor (Lima y Cunha, 2004).

Tratamiento IV (Autopolinización= A.P): Se marcaron y embolsaron botones florales (en bolsa de muselina $10 \times 20 \mathrm{~cm}$ ), se emascularon y polinizaron manualmente con la ayuda de un pincel con polen de la misma flor y se embolsaron nuevamente.

Tratamiento V (Geitonogamia= G): Se marcaron y embolsaron botones florales (en bolsa de muselina $10 \times 20 \mathrm{~cm}$ ), se emascularon y polinizaron manualmente con la ayuda de un pincel, con polen de otra flor de la misma planta y se embolsaron nuevamente.

En los tratamientos II, IV y V donde se realizó la polinización manual se distribuyó uniformemente la cantidad de polen transferida a cada flor con la ayuda de un pincel (Figura 1b), procurando una cantidad equitativa en cada uno de los estigmas de la flor. Por otro lado, para cada tratamiento se registró el porcentaje de fertilidad ocho días después de realizado el procedimiento. Cada uno constó de cuatro repeticiones en el tiempo y 25 botones por unidad experimental. Los frutos desarrollados fueron cosechados y caracterizados mediante las variables peso (gramos), dimensiones (longitud y diámetros en milímetros), porcentaje de pulpa (\%) y el número de semillas. Cabe anotar que cuando se presentaron días lluviosos los tratamientos de polinización no fueron realizados con el propósito de evitar sesgos en los valores obtenidos en la formación de frutos.

\section{Análisis de los datos}

Los datos obtenidos fueron tabulados en Excel 2010 (Microsoft $($ ) y analizados con el programa estadístico SAS v9. mediante parámetros univariados (promedio, desviación estándar y coeficiente de variación) y complementados con la prueba de comparación entre promedios de Duncan $(\mathrm{P}<0,05)$. Adicionalmente, esta información fue correlacionada con las variables climáticas: temperatura $\left({ }^{\circ} \mathrm{C}\right)$, radiación solar (h/día) y humedad relativa (HR) registradas durante el desarrollo del estudio.

\section{RESULTADOS Y DISCUSIÓN}

La polinización es una etapa crucial de la reproducción en la mayoría de las angiospermas, donde los vectores de polen son esenciales para mantener la transferencia de genes, tanto en ecosistemas naturales como agrícolas (Bonilla, 2012). Respecto a la formación de frutos, si hubo diferencias significativas entre los tratamientos de polinización natural (P.N), manual dirigida (P.M.D) y la autopolinización (A.P.E, G y A.P). La polinización natural por Xylocopa spp. presentó el mayor porcentaje promedio en la formación de frutos con un $88 \%$, superando en un $28 \%$ la polinización cruzada manual. Entre tanto, los trata- 
mientos de autopolinización espontánea (A.P.E) y los de autopolinización asistida (A.P, G) el porcentaje de formación de frutos varió entre 2,4 y $3,0 \%$, confirmando la alta alogamia de la especie y la importancia de los polinizadores (Figura 2). Los resultados concuerdan con lo hallado en estudios similares en Hawai y Estados Unidos por Akamine y Girolami (1959) y Knight y Winters (1962). El maracuyá es una de las especies que depende del servicio ecosistémico suministrado por los insectos en la polinización cruzada para la producción de frutos, debido a la presencia de

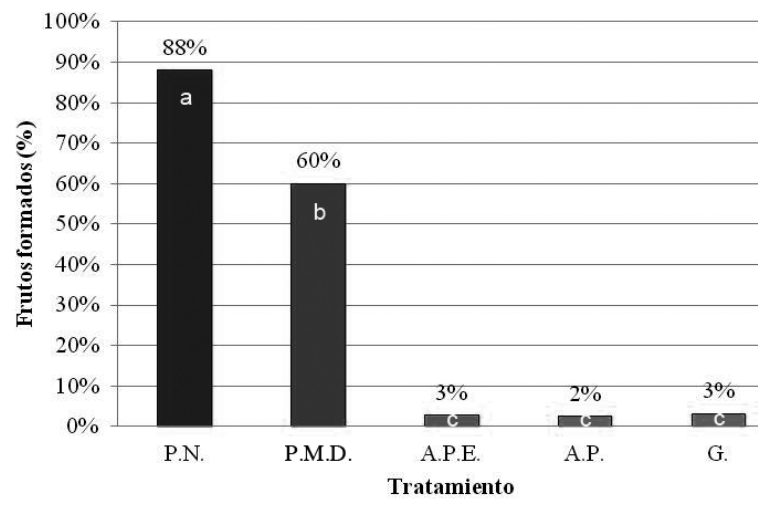

Figura 2. Porcentaje de frutos producidos bajo condiciones de campo en maracuyá $(n=100)$. P.N= Polinización natural, P.M.D= Polinización manual dirigida, A.P.E $=$ Autopolinización espontánea, A.P $=\mathrm{Au}-$ topolinización, $\mathrm{G}=$ Geitonogamia. Granja Luker. Colombia. 2010-2011. Letras diferentes indican diferencias significativas, Duncan $(\mathrm{P}<0,05)$. un sistema de autoincompatibilidad genética (Coppens d'Eeckenbrugge et al., 1997). A escala global, se estima que al menos un tercio de los cultivos dependen de los servicios de polinización proporcionada por insectos y otros animales para asegurar su producción (Calle et al., 2010; Goulson, 2010; Kjohl et al., 2011).

Los tratamientos de autopolinización presentaron altos coeficientes de variación (CV) entre 66 y $149 \%$ debido a que registraron valores extremos de cero (0) por el fenómeno de autoincompatibilidad (Cuadro 1). De acuerdo a Suassuna et al. (2000), en maracuyá este fenómeno es controlado por alelos esporofíticos (de $S_{1}$ a $S_{6}$ ) que actúan en asociación con genes gametofíticos los cuales limitan la fructificación. No obstante, las especies de Passiflora presentan características que favorecen la alogamia, como el tamaño de la flor, corona vistosa de color púrpura y néctar fragante y abundante (síndromes de polinización), las cuales están adaptadas a las necesidades de polinizadores especializados (Ramírez, 2006; Goulson, 2010). Estos síndromes son el resultado de la interacción mutualista entre la necesidad de la planta para movilizar su polen hasta los estigmas y proveer un recurso alimenticio para el polinizador (Bonilla, 2012).

La polinización natural presentó el menor coeficiente de variación $(11,13)$ lo que indica una mayor estabilidad cuando es realizada por los abejorros del género Xylocopa spp. En contraste, a pesar que con el tratamiento P.C se obtuvo un alto porcentaje de formación de frutos, este fue altamente variable (CV 31,41). La polinización en el maracuyá depende de un vector para el intercambio génico y la producción de frutos,

Cuadro 1. Rango de valores obtenidos para formación de frutos en maracuyá, en los tratamientos. Granja Luker. Colombia. 20102011.

\begin{tabular}{lcccccc}
\hline Tratamiento & $\begin{array}{c}\text { Mínimo } \\
(\boldsymbol{\%})\end{array}$ & $\begin{array}{c}\text { Máximo } \\
(\boldsymbol{\%})\end{array}$ & $\begin{array}{c}\text { Promedio } \\
(\boldsymbol{\%})\end{array}$ & Varianza & Des. Est. & CV $(\boldsymbol{\%})$ \\
\hline Polinización natural (P.N) & 76 & 100 & $88,0 \mathrm{a}$ & 96,8 & 9,84 & 11,13 \\
Polinización manual dirigida (P.M.D) & 35 & 85 & $60,0 \mathrm{~b}$ & 352,7 & 18,78 & 31,41 \\
Autopolinización espontánea (A.P.E) & 0 & 4 & $2,7 \mathrm{c}$ & 4,3 & 2,07 & 77,46 \\
Autopolinización (A.P.) & 0 & 8 & $2,4 \mathrm{c}$ & 12,8 & 3,58 & 149,00 \\
Geitonogamia (G) & 0 & 4 & $3,0 \mathrm{c}$ & 4,0 & 2,00 & 66,67 \\
\hline
\end{tabular}

Letras diferentes indican diferencias significativas, Duncan $(\mathrm{P}<0,05)$.

$\mathrm{CV}=$ Coeficiente de variación . 
similar a lo reportado por otros autores (Da Silva et al., 1999; Caicedo et al., 1993a). En la zona de estudio, se observaron insectos de Xylocopa spp. como responsables de la polinización, los cuales fueron identificados con la ayuda de las claves de González et al. (2009) y los criterios de experto. La frecuencia de visitas observadas de estos polinizadores durante el periodo de antesis fue de ocho para cada flor. Estas observaciones establecen que su abundancia durante este estudio fue alta, acorde a lo publicado por Da Silva et al. (1999), donde indican que una frecuencia de visitas mayor o igual a 7,2 son suficientes para obtener porcentajes de fructificación superiores a $80 \%$. Aunque, Apis mellifera puede ser un visitante ocasional, Peña (2003) menciona que esta solo tiene un 3\% de efectividad en la formación de frutos, por lo cual no debe ser considerado como un vector para la especie. Por tal motivo, se puede indicar que la mayor eficacia de los abejorros (Xylocopa spp.) frente a la polinización manual, se debió probablemente a las altas poblaciones presentes en la zona de estudio y a la alta especiación con el maracuyá, tal como se ha estudiado en otras especies de Passiflora (Ramírez 2006). En la planeación de las actividades agronómicas se debe tener en cuenta la presencia de los polinizadores para asegurar la producción de frutas, así como el valor económico de su servicio (Calle et al., 2010; Medina-Gutiérrez et al., 2012).

Hasta hace poco tiempo casi todos los agricultores consideraban la polinización como uno de los numerosos "servicios gratuitos" de la naturaleza, al punto de clasificarse muy pocas veces como "insumo agrícola" o como un tema de relevancia para ser tratado. Sin embargo, en la actualidad esta perspectiva ha cambiado, debido a la disminución hasta del $96 \%$ en su abundancia relativa, según lo demuestran estudios publicados en Gran Bretaña y Holanda por Biesmeijer et al. (2006) y en Estados Unidos por Cameron et al. (2011).

En países productores de maracuyá como Brasil, la eficiencia de la polinización natural no sobrepasa el 12\% (Bos et al., 2007; Siqueira et al., 2009), debido probablemente a la fragmentación, pérdida de hábitats, intensificación y uso inadecuado de agroquímicos (Calle et al., 2010). Estas actividades han puesto en peligro el servicio de polinización natural en los cultivos y a su vez afectado el mantenimiento de la diversidad y la abundancia de estos (Kremen et al.,
2004). En Colombia, Calle et al. (2010) mencionan que la polinización manual es necesaria cuando existe una zona especializada en el cultivo y la percepción de los productores de mantener la conservación de las áreas de bosque que albergan a los polinizadores.

La utilización de la polinización manual para contrarrestar esta limitante surge como una estrategia para aumentar la producción en zonas altamente perturbadas (Da Silva et al., 1999). De acuerdo con Ishihata (1991), en aquellos países donde no existen los polinizadores naturales, este tipo de polinización es empleada con gran éxito. Varios estudios mencionan una eficiencia entre 50 y $87 \%$ en la formación de frutos con esta metodología (Bos et al., 2007; Siqueira et al., 2009; Rendón et al., 2014), además los valores obtenidos en esta investigación coinciden con estudios anteriores $(\mathrm{PC}=60 \%)$.

En cuanto al número de semillas por fruto, se observaron diferencias significativas entre tratamientos, donde la polinización natural (P.N) y manual dirigida (P.M.D) registraron los mayores valores, 350 y 250 respectivamente (Figura 3). Los tratamientos de polinización espontánea (A.P.E), autopolinización (A.P) y geitonogamia (G) mostraron un comportamiento progresivo en el número de semillas por fruto $(35,98$ y 148), lo cual sugiere que el aumento de granos de polen sobre los estigmas de la flor polinizada aumentó la probabilidad de óvulos fecundados. De acuerdo a

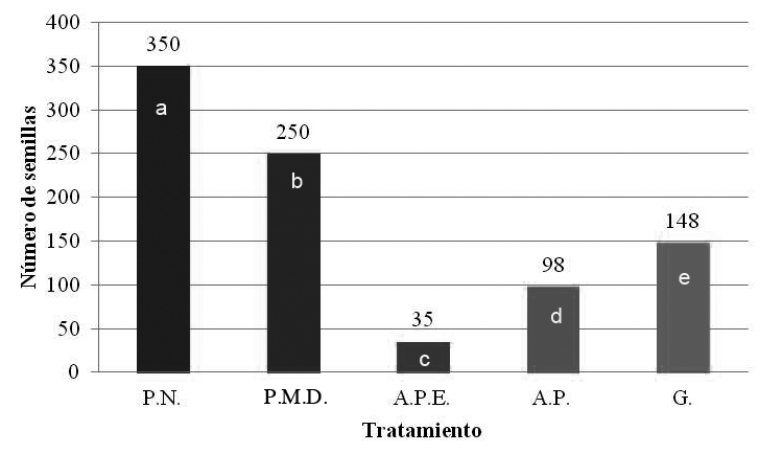

Figura 3. Número de semillas por fruto obtenidos en los diferentes tratamientos de polinización en maracuyá. P.N= Polinización Natural, P.M.D= Polinización manual dirigida, A.P.E $=$ Autopolinización Espontánea, A.P= Autopolinización, $G=$ Geitonogamia. Granja Luker. Colombia. 20102011. Letras diferentes indican diferencias significativas, Duncan $(\mathrm{P}<0,05)$. 
Silveira et al. (2012), el número de semillas por fruto es un parámetro importante que debe ser considerado en los estudios que involucren diferentes tipos de polinización. Estos resultados confirman que la tasa de autofecundación en el maracuyá puede aumentar con la presencia de un vector animado, debido a que los valores obtenidos en esta investigación se incrementaron hasta un $77 \%$. Un estudio realizado en Venezuela menciona que el número promedio de óvulos en maracuyá fue de 264 por ovario y solo el 72\% (191) forma semilla vía polinización natural (Aponte y Jáuregui, 2004). Estos valores difieren con los resultados obtenidos en este estudio, debido a que el número de semillas promedio por fruto fue de 350 .

Por otro lado, no todos los frutos formados en los tratamientos de autopolinización (A.P.E, A.P, G) alcanzaron la madurez fisiológica, por lo cual, la caracterización física de los frutos solo se realizó para la polinización natural y para la manual dirigida, con un total de 40 y 30 frutos (Cuadro 2), respectivamente. La polinización P.N mostró un incremento en las variables peso promedio del fruto (8\%), porcentaje de pulpa más semilla (6\%) y el porcentaje de pulpa $(2,3 \%)$ en comparación a la polinización cruzada manual. Además, los coeficientes de variación promedio $(\mathrm{CV})$ en todas las variables de la polinización manual (P.M.D, 18,82\%) fueron superiores a la P.N $(11,88 \%)$, indicando una mayor estabilidad cuando interviene el abejorro polinizador (Cuadro 2). El efecto positivo de la polinización natural sobre variables de calidad como peso $(203,1 \mathrm{~g})$ y porcentaje de pulpa $(47,0 \%)$, concuerda con las observaciones de Ruggiero et al. (1996) en Brasil, los cuales indican que estas características están directamente relacionadas a un buen proceso de polinización; además, coinciden con lo mencionado por Angel-Coca et al. (2011) para P. edulis f. edulis. En este sentido, Silveira et al. (2012) indica que la aplicación de diferentes tratamientos de polinización no altera características físicas del fruto, como peso, longitud y diámetro.

El aumento en las características del fruto se debe probablemente a que las flores están expuestas a numerosas visitas por parte de los polinizadores, los cuales en su proceso de recolección pueden llevar en el dorso polen de hasta 25 flores y consecuentemente aumenta la fecundación (Janzen, 1968), tardando entre 5,4 a 8,5 s por visita (Caicedo et al., 1993a; Peña, 2003). Estos últimos autores indican que un solo individuo de Xylocopa puede visitar más de 1800 flores en un periodo inferior a tres horas. En el caso de la polinización cruzada manual, Caicedo et al. (1993a) resaltan que el número de estructuras visitadas por día disminuyó en un $46 \%$ en comparación al polinizador natural. Ángel-Coca et al. (2011) llegaron a esta misma conclusión en ensayos realizados en la gulupa $(P$. edulis f. edulis). En contraste, Ocampo et al. (2012) describen un mayor número de semillas cuando se realiza la polinización manual en la gulupa. Sin embargo, las diferencias entre estos resultados dependerán de la cantidad y actividad de los polinizadores en cada zona agroecológica donde los estudios fueron realizados.

La mayor eficiencia de la polinización natural se debió posiblemente a las altas poblaciones de abejorros de Xylocopa spp. en la zona, y a su vez por la gran diversidad de especies de pasifloras sembradas en los alrededores de la colección. En este sentido, Akamine et al. (1954) indica que una forma de incrementar las poblaciones de polinizadores en una zona específica,

Cuadro 2. Características relacionadas con el fruto, peso y semilla de la polinización natural y manual de maracuyá. Granja Luker. Colombia. 2010-2011.

\begin{tabular}{|c|c|c|c|c|c|c|}
\hline \multirow[t]{2}{*}{$\begin{array}{l}\text { Características de } \\
\text { los frutos }\end{array}$} & \multicolumn{3}{|c|}{$\begin{array}{l}\text { Polinización natural } \\
\qquad(\mathrm{n}=40)\end{array}$} & \multicolumn{3}{|c|}{$\begin{array}{l}\text { Polinización manual dirigida } \\
\qquad(\mathrm{n}=\mathbf{3 0})\end{array}$} \\
\hline & Promedio & Des. Est. & $\mathrm{CV}(\%)$ & Promedio & Des. Est. & $\mathrm{CV}(\%)$ \\
\hline Longitud (mm) & 91,6 & 6,92 & 7,6 & 89,6 & 10,1 & 11,2 \\
\hline Diámetro $(\mathrm{mm})$ & 76,9 & 4,59 & 6,0 & 74,5 & 7,0 & 9,4 \\
\hline Peso total (g) & 203,1 & 37,45 & 18,4 & 187,5 & 49,7 & 26,5 \\
\hline Peso pulpa + semilla $(\mathrm{g})$ & 94,7 & 17,23 & 18,2 & 84,5 & 26,6 & 31,5 \\
\hline Porcentaje de pulpa (\%) & 47,0 & 4,32 & 9,2 & 44,7 & 7,0 & 15,7 \\
\hline
\end{tabular}


se basa en el aumento de las fuentes de polen y néctar en el momento de la floración del maracuyá. La cantidad de hábitat natural disponible cerca de áreas cultivadas, puede afectar notablemente la prestación del servicio de polinización por los insectos nativos (Bonilla, 2012).

Las relaciones de las variables climáticas de temperatura máxima diurna $\left(\operatorname{Tmax}^{\circ} \mathrm{C}\right)$, humedad relativa (HR) y brillo solar (BS) con la P.N se presentan en la Figura 4. Hubo correlaciones positivas entre el porcentaje de formación de frutos y las variables evaluadas, con valores de $r=0,69\left(\operatorname{Tmax}^{\circ} \mathrm{C}\right) ; r=0,64$ (HR)
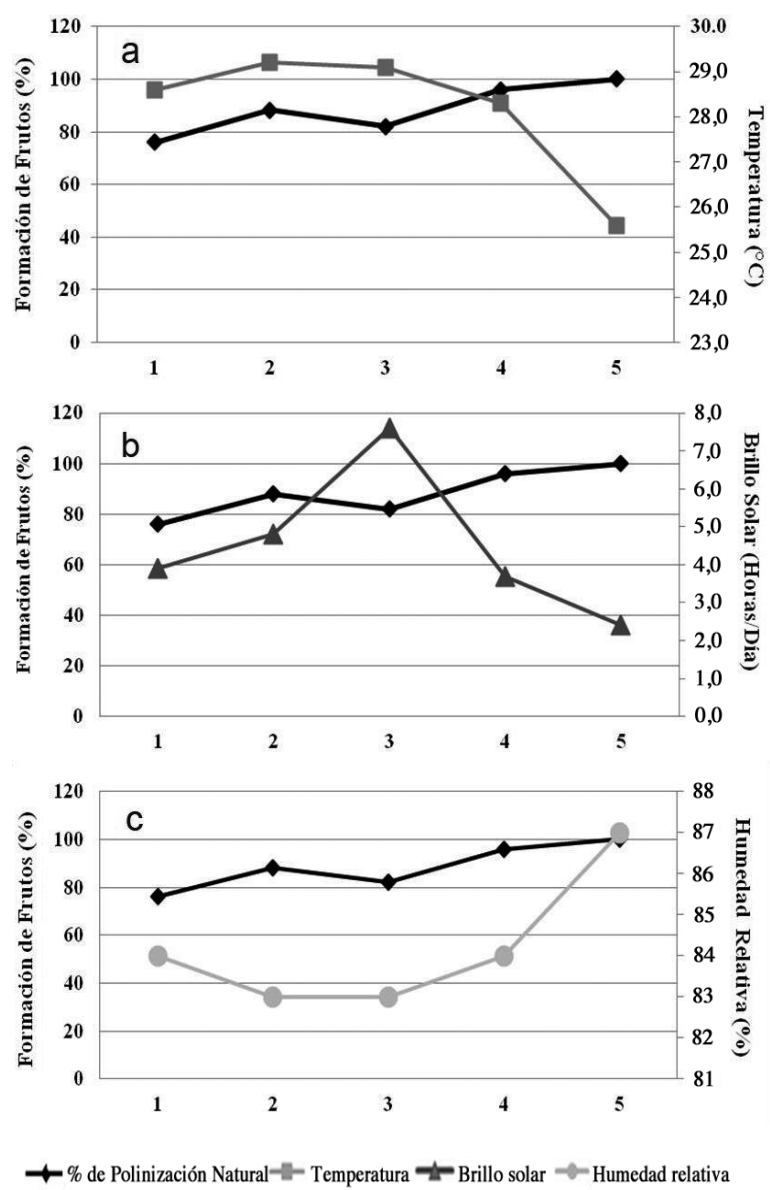

Figura 4. Relación entre el porcentaje de frutos formados por polinización natural (P.N) en maracuyá y temperatura $\left({ }^{\circ} \mathrm{C}\right)(\mathrm{a})$, brillo solar (h/día) (b) y humedad relativa (\%) (c). Granja Luker. Colombia. 20102011. y $r=0,56$ (BS). Esto indica que en la mayoría de las repeticiones existió un comportamiento directamente proporcional con la formación de frutos. De acuerdo con Corbet y Willmer (1980), durante los días nublados, el porcentaje de formación de frutos fue en promedio del $25 \%$, mientras que se puede alcanzar hasta un $94 \%$ en días soleados. Así mismo, Da Silva et al. (1999) indican que el aumento de la humedad relativa (55 a 80\%) genera una reducción en la actividad de los abejorros polinizadores del género Xylocopa. Además, estos factores climáticos también pueden influir en la germinación del polen (Free, 1993). En contraste, las condiciones de baja temperatura $\left(25,6^{\circ} \mathrm{C}\right)$, brillo solar $(2,4 \mathrm{~h} /$ día $)$ y una humedad relativa $(\geq 80 \%)$ pueden incrementar el porcentaje de frutos formados.

Los polinizadores muestran alta sensibilidad a los cambios de temperatura y radiación solar, debido posiblemente a los límites en la capacidad de control de la temperatura corporal de estos insectos (Da Silva et al., 1999). De igual manera, estos autores mencionan que el aumento de temperatura superior a $26^{\circ} \mathrm{C}$, reduce la densidad de los polinizadores (Xylocopa spp.) y el número de frutos formados de maracuyá. Esta observación, coincide con lo encontrado en este estudio, ya que la máxima tasa de fructificación por polinización natural se presentó con una temperatura de $25,6^{\circ} \mathrm{C}$ (Figura 4). En este sentido, Fischer et al. (2009) menciona que temperaturas entre 20 y $25^{\circ} \mathrm{C}$ son ideales para fomentar la actividad de los polinizadores y por consiguiente, el número de flores polinizadas. En relación con lo anterior, el componente ambiental tiene un efecto notorio sobre la interacción planta/polinizador, debido a que la fenología floral puede alterarse por los cambios climáticos extremos e intervenir en el acople temporal con los insectos polinizadores (Hegland et al., 2009).

La precipitación tuvo un efecto negativo sobre la producción de frutos, debido a que disminuyó la actividad de los polinizadores antes y durante la antesis (+/- entre las 10 am y 5 pm). En relación con lo anterior, la receptividad estigmática y viabilidad del polen estuvieron directamente relacionadas con valores máximos de las variables climáticas registradas en este estudio.

En cuanto a las estrategias para fomentar e incrementar las poblaciones de Xylocopa spp. se sugiere la ubicación de sitios donde puedan construir los nidos, como postes de madera roja y liviana (Akamine et al., 
1954; Free, 1993). En Colombia, el sustrato preferido por Xylocopa en un cultivo de maracuyá es la madera en proceso de descomposición, así como troncos viejos de plantas de cacao (Theobroma cacao L.) (Caicedo et al., 1993b). Los sustratos preferidos por X. frontalis, y el mataratón (Gliricidia sepium (Jacq.) Kunth ex Walpers) por X. aenipennis en Viterbo (Caldas, Colombia) son: el cedro (Cedrella montana Turz.), la guayaba (Psidium guajava L.) y el guamo (Inga edulis Mart.) (Peláez, 2004) . En Brasil, Camillo (1996) incrementó la producción de frutos en maracuyá de 3,2 a $25 \%$ al introducir postes con nidos de los abejorros, $X$. frontalis y $X$. grisescens. Cultivos de maracuyá donde el grado de aislamiento del hábitat natural/semi-natural es bajo, dependen exclusivamente de los polinizadores naturales (Xylocopa spp.) para la producción de frutos, sin la necesidad de recurrir a la polinización manual (Calle et al., 2010). Así mismo, estos autores sugieren que la eliminación del uso de herbicidas alrededor de los cultivos sería suficiente para promover la presencia de estas especies. En general, las intervenciones y/o estrategias a implementar en el contexto de la provisión de servicios de polinización natural, dependerán de las condiciones de cada agropaisaje en particular.

Los polinizadores naturales proporcionan un servicio ambiental esencial tanto para los ecosistemas naturales como para los agroecosistemas. En el caso del maracuyá, los resultados aquí descritos denotan el papel ecológico que cumplen tanto desde el punto de vista productivo como en el mantenimiento de la diversidad genética. Estos componentes deben ser integrados a los planes de manejo del cultivo, donde la implementación de buenas prácticas agrícolas (BPA) contribuya en su conservación, debido a que la producción y calidad de frutos depende de ellos. Además, los agricultores y comunidades deben adoptar medidas que busquen conservar y fortalecer los lazos entre los diferentes aspectos de la agrobiodiversidad, y contribuir a la estabilidad a largo plazo frente al cambio climático inminente como ya vienen estudiando en Brasil (Giannini et al., 2013).

\section{AGRADECIMIENTOS}

Los autores agradecen al Ministerio de Agricultura y Desarrollo Rural (MADR) por la financiación del proyecto "Aprovechamiento de la diversidad del maracuyá, la gulupa y la granadilla para mejorar y diversificar los sistemas de producción en Colombia"; al Centro de Bio-Sistemas de la Universidad Jorge Tadeo por la coordinación, a CASA LUKER, por el establecimiento y mantenimiento de la colección y a todas aquellas personas que contribuyeron en el desarrollo de la investigación.

\section{LITERATURA CITADA}

Agronet. 2012. Ministerio de Agricultura y Desarrollo Rural de Colombia. Análisis - estadísticas, maracuyá. http:// www.agronet.gov.co (Consultado 5 nov. 2012).

Akamine, E.K., R.A. Hamilton, T. Nishida, G.D. Sherman, y W.B. Storey. 1954. Passion fruit culture. University of Hawaii, US.

Akamine, E.K., y G. Girolami. 1959. Pollination and fruit set in the yellow passion fruit. Agricultural Experiment Station and Extension Technical Bulletin. Hawaii, USA.

Ángel-Coca, C., G. Nates, R. Ospina, C.D. Melo, y M. Amaya. 2011. Biología floral y reproductiva de la gulupa Passiflora edulis Sims f. edulis. Caldasia 33(2):433-451.

Aponte, Y., y D. Jauregui. 2004. Capacidad reproductiva: formación de frutos y semillas en Passiflora edulis $\mathrm{f}$. flavicarpa Degener y Passiflora cincinnata Mast. Rev. Fac. Agro. (LUZ) 21:353-361.

Biesmeijer, J.C., S.P.M. Robert, y M. Reemer. 2006. Parallel declines of pollinators and insect - pollinated plants in Britain and the Netherlands. Science 313:351-354.

Bonilla, M.A. 2012. La polinización como servicio ecosistémico. En: Iniciativa colombiana de polinizadores (ICPA), Capítulo I: abejas. Universidad Nacional de Colombia, Instituto Humboldt. Bogotá, Colombia. 103 p.

Bos, M.M., D. Veddeler, A.K. Bogdanski, A. Klein, T. Tscharntke, I. Steffan-Dewenter, y J.M. Tylianakis. 2007. Caveats to quantifying ecosystem services: fruit abortion blurs benefits from crop pollination. Ecol. Applic. 17(6):1841-1849.

Caicedo, G., H. Vargas, y M. Gaviria. 1993a. Evaluación de Xylocopa spp. (Hymenoptera: Anthophoridae) como polinizadores en el cultivo de maracuyá (Passiflora edulis var. flavicarpa Degener). Rev. Col. Entomol. 19(3):107-110.

Caicedo, G., H. Vargas, y M. Gaviria. 1993b. Estudio del modelo natural de asentamiento en Xylocopa 
(Hymenoptera: Anthophoridae) para la adaptación de refugios en el cultivo de maracuyá (Passiflora edulis var. flavicarpa Degener). Revista Colombiana de Entomología 19(2):72-78.

Calle, Z., M.R. Guariguata, E. Giraldo, y J. Chará. 2010. La producción de maracuyá (Passiflora edulis) en Colombia: perspectivas para la conservación del hábitat a través del servicio de polinización. Interciencia 35(3):207-212.

Cameron, S.A., J.S. Loziera, J.P. Strangeb, J.B. Kochb, N. Cordesa, L.F. Solterd, y T.L. Griswold. 2011. Patterns of widespread decline in North American bumble bees. PNAS 108(2):662-667.

Camillo, E. 1996. Utilização de espécies de Xylocopa (Hymenoptera: Anthophoridae) na polinização do maracujá amarelo. En: Anais do Encontro Sobre Abelhas. Ribeirão Preto, Brasil. p. 141-146.

Coppens d'Eeckenbrugge, G., S. Segura, E. Hodson de Jaramillo, y G. Góngora. 1997. Les fruits de la passion. En: A. Charrier, M. Jacquot, S. Hamon, y D. Nicolas, editores, L'amélioration des plantes tropicales. CIRAD-ORSTOM, Repères, Montpellier, Francia. p. 291-312.

Corbet, S.A., y P.G. Willmer. 1980. Pollination of the yellow passion fruit: nectar, pollen y carpenter bees. Journal of Agricultural Science 95:655-666.

Da Silva, M., C. Bruckner, M. Picanco, y A. Molina. 1999. Número floral, clima, densidad poblacional de Xylocopa spp. (Hymenoptera: Anthophoridae) y polinización del maracuyá (Passiflora edulis f. flavicarpa). Rev. Biol. Trop. 47(4):711-718.

Fischer, G., F. Casierra-Posada, y W. Piedrahíta. 2009. Ecofisiología de las especies pasifloráceas cultivadas en Colombia. En: D. Miranda, G. Fischer, C. Carranza, S. Magnitskiy, F. Casierra, W. Piedrahíta, y L.E. Flórez, editores, Cultivo, poscosecha y comercialización de las pasifloráceas en Colombia: maracuyá, granadilla, gulupa y curuba. Sociedad Colombiana de Ciencias Hortícolas. Bogotá, Colombia. p. 45-67.

Free, J.B. 1993. Insect pollination of crops. 2 ed. Academic Press. London, United Kingdom.

Ghazoul, J. 2007. Challenges to the uptake of the ecosystem service rationale for conservation. Conservation Biology 21:1651-1652.

Giannini,T.C.,A.L.Acosta,C.I.Da Silva,P.E.A.Oliveira, V.L. Imperatriz-Fonseca, y A.M. Saraiva. 2013. Identifying the areas to preserve passion fruit pollination service in Brazilian Tropical Savannas under climate change. Agric. Ecosyst. Environ. 171: 39-46.
González, V.H., M.M. González, y Y. Cuellar. 2009. Notas biológicas y taxonómicas sobre los abejorros del maracuyá del género Xylocopa (Hymenoptera: Apidae, Xylocopini) en Colombia. Acta Biol. Col. $14(2): 31-40$.

Goulson, D. 2010. Bumblebees: behaviour, ecology and conservation. 2 ed. Oxford University Press, New York, United States.

Hegland, S.J., A. Nielsen, A. Lazaro, A.L. Bjerknes, y O. Totland. 2009. How does climate warming affect plant - pollinator interactions?. Ecology Letters 12(2): 184-195.

Ishihata, K. 1991. Studies on pollen germination and tube growth from normal and upright style flowers in purple passion fruit (Passiflora edulis Sims) using various artificial media. Japanese Journal of Tropical Agriculture 35(2):98-103.

Janzen, D.H. 1968. Reproductive behavior in the Passifloraceae and some of its pollinators in Central América. Behavior 32:33-48.

Jaramillo, J., J. Cárdenas, y J. Orozco. 2009. Manual sobre el cultivo del maracuyá (Passiflora edulis) en Colombia. Corpoica, Palmira, Colombia.

Kjohl, M., A. Nielsen, y C. Stenseth. 2011. Potential effects of climate change on crop pollination. FAO, Rome, Italy.

Kremen, C., N.M. Williams, R.L. Bugg, J.P. Fay, and R.W Thorp. 2004. The area requirements of an ecosystem service: crop pollination by native bees communities in California. Ecology letters 7:1109-1119.

Knight, R.J., y H.F. Winters. 1962. Pollination and fruit set of yellow passionfruit in Southern Florida. Florida State Horticultural Society. p. 412-418.

Lima, Ade A., y M.A.P. Cunha. 2004. Da maracujá: producao e qualidade na passicultura. Embrapa Mandioca e Fruticultura. Cruz de Almas, Brasil.

Medina-Gutiérrez, J., R. Ospina-Torres, y G. Nates-Parra. 2012. Efectos de la variación altitudinal sobre la polinización en cultivos de gulupa (Passiflora edulis f. edulis). Acta Biol. Col. 7(2):379-394.

Ocampo, J., D. Melo, J. Rendón, J.C. Arias, y V. Marín. 2012. Aspectos fisiológicos de la gulupa. p. 13-15. En: J. Ocampo, y K. Wyckhuys, editores, Tecnología para el cultivo de la gulupa (Passiflora edulis f. edulis Sims) en Colombia. Centro de Bio-sistemas Universidad Jorge Tadeo Lozano, Centro Internacional de Agricultura Tropical CIAT y MADR. Bogotá, Colombia. 68 p.

Passionfruit. 2011. Supply and demand. http://www. passionfruitjuice.com (Consultado 3 oct. 2011). 
Peláez, J.M. 2004. Recursos florales usados por Xylocopa frontalis en el Valle del Risaralda, Colombia. [CD]. En: Memorias del Encuentro Colombiano sobre Abejas Silvestres. Colombia. p. 90-100.

Peña, J.E. 2003. Insectos polinizadores de frutas tropicales: no solo las abejas llevan miel al panal. Manejo Integrado de Plagas y Agroecología. Costa Rica 69:6-20.

Ramírez, W. 2006. Hibridación interespecífica en Passiflora (Passiflorácea), mediante polinización manual, y características florales para la polinización. Lankesteriana 6(3):123-131.

Rendón, J.S., J. Ocampo, y R. Urrea. 2013. Estudio sobre la polinización y biología floral en Passiflora edulis f. edulis Sims, como base para el prejoramiento genético. Acta Agronómica 62(3):232-241.

Roubik, D.W. 1995. Pollination of cultivated plants in the tropics. FAO, Rome, Italy.

Ruggiero, C., A.R. Sao José, C.A. Volpe, J.C. Oliveira, J.G. Durigan, J.R. Baumgartner, K. Silva, M.E. Nakamura,
R. Ferreira, R. Kavati, y V.P. Pereira. 1996. Maracujá para exportacão: aspectos técnicos da producão. Ministerio da Agricultura e do Abastecimento, Secretaria do Desemvolvimento Rural, Programa do Apoio a Producão e Exportacão de Frutas, Hortalicas, flores e Plantas Ornamentais. Brasilia: EMBRAPA SPI, Brasil.

Silveira, M.V., A.R. Abot, J.N. Nascimento, E.T. Rodrigues, S.R. Rodrigues, y A. Puker. 2012. Is manual pollination of yellow passion fruit completely dispensable?. Sci. Hortic. 146:99-103.

Siqueira, K., L. Kill, C.F. Martins, I.B. Lemos, S.P. Monteiro, y E. de A. Feitoza. 2009. Ecología da polinizacão do maracuja-amarelo, na região do vale do sunmedio São Francisco. Rev. Bras. Frutic. 31(1):001-012.

Suassuna, T.M.F., C.H. Bruckner, C.R. Carvalho, y A. Borém. 2000. Self-incompatibility in passionfruit: evidence of gametophytic - sporophytic control. Theor. Appl. Genet. 106:298-302. 